Pacific Journal of Mathematic 


\section{PRINCIPAL IDEAL AND NOETHERIAN GROUPS}

\section{S. Feigelstock and Z. Schlussel}

Let $\Pi$ be a ring property. An additive group $G$ is said to be an (associative) strongly $\Pi$-group if $G$ is not nil, and if every (associative) ring $R$ with additive group $G$ such that $R$ is not a zeroring has property $\Pi$. The (associative) strongly principal ideal groups, and the (associative) strongly Noetherian groups are classified for groups which are not torsion free. Some results are also obtained for the torsion free case.

(i) All groups considered here are abelian, with addition the group operation. Rings are not necessarily associative.

Let $\pi$ be a ring property. A group $G$ is said to be an (associative) $\pi$-group, denoted by $(A) \pi$-group, if there exists an (associative) ring $R$ with additive group $G$ such that $R$ is not a zeroring, and $R$ has property $\pi$. $G$ is an (associative) strongly $\pi$-group, denoted by $(A) S \pi$-group. if $G$ is an $(A) \pi$-group, and every (associative) ring with additive group $G$ which is not the zeroring on $G$ has property $\pi$.

If the only (associative) ring with additive group $G$ is the zeroring, then $G$ is said to be an (associative) nil group, denoted by $(A)$ nil group.

The two ring properties $\pi$ considered in this paper are:

1. every two-sided ideal is principal, denoted by $P I$,

2. every two-sided ideal is finitely generated, denoted by $N$.

In (ii) a complete characterization of the torsion $(A) S P I$ groups will be given. It will be shown that there are no mixed $(A) S P I$ groups. Some results concerning torsion free $(A) S P I$ groups will be obtained. In (iii), the torsion, and mixed $S N$ groups will be completely characterized. Some results concerning torsion free $S N$ groups will be given.

(ii) If $X$ is a nonempty subset of a group or ring, $(X)$ denotes the additive subgroup generated by $X$, and $\langle X\rangle$ denotes the ideal generated by $X$.

If $G=G_{1} \oplus G_{2}$ is a group, $\pi_{G_{i}}$ is the natural projection of $G$ on $G_{\imath}$, for $i=1,2$.

Lemma 1. Let $G=H \oplus K, H \neq 0, K \neq 0$, be an ASPI-group. Then $H$ and $K$ are either both cyclic or both $A$ nil. 
Proof. Suppose that $H$ is not $A$ nil. Let $S$ be an associative ring on $H$ which is not the zeroring on $H$, and let $T$ be the zeroring on $K$. The ring direct sum $R=S \oplus T$ is an associative ring on $G$, which is not the zeroring on $G$. $T$ is an ideal in $R$, and hence $T=\langle x\rangle$. Clearly $K=(x)$. Therefore $K$ is not $A$ nil. The above argument, interchanging the roles of $H$ and $K$, yields that $H$ is cyclic.

Corollary. Let $G=H \oplus K, H \neq 0, K \neq 0$ be an SPI-group. Then $H$ and $K$ are cyclic.

Proof. It suffices to negate that $H$ and $K$ are both $A$ nil. Suppose this is so. Let $R$ be a ring on $G$ which is not the zeroring on $G$.

(1) Suppose that $R^{2} \subseteq K$. There exist $h_{0} \in H, k_{0} \in K$ such that $R=\left\langle h_{0}+k_{0}\right\rangle$. Let $h \in H$. Since $h \in R$, there exists an integer $n$, and $x \in R^{2}$ such that $h=n\left(h_{0}+k_{0}\right)+x$. However, $x \in K$. Hence $h=n h_{0}$, and $H$ is cyclic, contradicting the fact that $H$ is $A$ nil.

(2) Suppose that $R^{2} \not \subset K$. For all $g_{1}, g_{2} \in G$, define $g_{1} \times g_{2}=$ $\pi_{H}\left(g_{1} g_{2}\right)$. Then $S=(G, \times)$ is a not necessarily associative ring on $G$, which is not the zeroring on $G$, satisfying $S^{2} \subseteq H$. The argument employed in (1) yields that $K$ is cyclic, contradicting the fact that $K$ is $A$ nil.

THEOREM 1. Let $G$ be a nonzero torsion group. The following are equivalent:

(1) Either $G$ is cyclic, or $G \simeq Z(p) \oplus Z(p)$ for a prime $p$.

(2) $G$ is $S P I$.

(3) $G$ is ASPI.

Proof. (1) $\Rightarrow(2)$ : Nontrivial cyclic groups are clearly SPI. Suppose that $G=\left(x_{1}\right) \oplus\left(x_{2}\right)$ with $\left|x_{i}\right|=p$ a prime, $i=1,2$. Let $R$ be a ring on $G$ which is not the zeroring on $G$, and let $l$ be a proper ideal in $R$. Then $|l|=0$ or $p$, and hence $l$ is generated by a single element. It therefore suffices to show that $R$ is generated by a single element. We may assume that $R \neq\left\langle x_{1}\right\rangle$, and that $R \neq\left\langle x_{2}\right\rangle$. Hence $\left\langle x_{i}\right\rangle=\left(x_{i}\right)$ for $i=1,2$. This implies that

$$
x_{i} x_{j}= \begin{cases}k_{i} x_{i}, 0<k_{i}<p, & \text { if } i=j, i=1,2 \\ 0 & \text { if } i \neq j, i, j=1,2 .\end{cases}
$$

Put $l=\left\langle x_{1}+x_{2}\right\rangle$. Let $r, s$ be integers such that $r k_{1}+s p=1$. Then $r x_{1}\left(x_{1}+x_{2}\right)=r k_{1} x_{1}=(1-s p) x_{1}=x_{1}$. Hence $x_{1} \in l$, and so $\left(x_{1}+x_{2}\right)-$ $x_{1}=x_{2} \in l$. Therefore $l=R$.

(2) $\Rightarrow$ (3): Let $G$ be a torsion SPI group. It suffices to show that $G$ 
admits an associative, nonzero multiplication. If $G$ is indecomposable then $G \simeq Z\left(p^{n}\right), p$ a prime, and $n$ a positive integer or $\infty$ [1, Corollary 27.4]. If $n=\infty$ then $G$ is nil. If $n$ is a positive integer then $G$ admits a ring structure is isomorphic to $Z_{p^{n}}$ the ring of integers modulo $p^{n}$. If $G$ is decomposable, then by the Corollary to Lemma $1, G \simeq$ $Z(n) \oplus Z(m)$. Hence $G$ admits a ring structure isomorphic to the ring direct sum $Z_{n} \oplus Z_{m}$.

(3) $\Rightarrow(1)$ : Suppose that $G$ is ASPI. If $G$ is indecomposable, then $G \simeq Z\left(p^{k}\right), p$ a prime, $1 \leqq k \leq \infty,[1$, Corollary 27.4]. However $Z(p \infty)$ is $A$ nil [ 3 , Satz 1 , and Zusatz]. Hence $G$ is cyclic. By Lemma 1 and [1, Theorem 120.3] we may assume $G=\left(x_{1}\right) \oplus\left(x_{2}\right)$ with $\left|x_{l}\right|=n_{\imath}, i=$ 1,2. If $\left(n_{1}, n_{2}\right)=1, G$ is cyclic; otherwise, let $p$ be a prime divisor of $\left(n_{1}, n_{2}\right)$. Then $G=\left(y_{1}\right) \oplus\left(y_{2}\right) \oplus H$, with $\left|y_{i}\right|=p^{m_{1}}, i=1,2$, and $1 \leqq m_{1} \leqq$ $m_{2}$. Since $\left(y_{1}\right) \bigoplus\left(y_{2}\right)$ is neither cyclic nor $A$ nil, $H=0$ by Lemma 1 .

Now let $R$ be the ring on $G$ with multiplication defined by $y_{i} y_{j}=p^{m_{2}-1} y_{2}$ for $i, j=1,2$. Then $R$ is an associative ring on $G$ which is not a zeroring, so $R=\left\langle s_{1} y_{1}+s_{2} y_{2}\right\rangle$ for some $s_{1}, s_{2} \in Z$. Every $x \in R$ has the form $x=k_{x} s_{1} y_{1}+\left(k_{x} s_{2}+m_{x} p^{m_{2}-1}\right) y_{2}$, for some $k_{x}, m_{x} \in Z$. In particular, $y_{1}=k_{y_{1}} s_{1} y_{1}$, and $y_{2}=\left(k_{y_{2}} s_{2}+m_{y_{2}} p^{m_{2}-1}\right) y_{2}$. Hence if $m_{2}>1, k_{y_{1}} s_{1} \equiv$ $1(\bmod p), k_{y_{2}} s_{2}+m_{y_{2}} p^{m_{2}-1} \equiv 1(\bmod p)$, so $p \nmid k_{y_{1}}$ and $p \nmid s_{2}$. However $k_{y_{1}} s_{2}+m_{y_{1}} p^{m_{2}-1} \equiv 0(\bmod p)$, so that either $p \mid k_{y_{1}}$, or $p \mid s_{2}$. This is a contradiction, so $m_{2}=1=m_{1}$.

\section{THEOREM 2. There are no mixed ASPI-groups.}

Proof. Let $G$ be a mixed $A S P I$-group. $G$ is decomposable, [1, Corollary 27.4], so by Lemma $1, G=H \oplus K, H \neq 0, K \neq 0$, with $H$ and $K$ either both cyclic or both $A$ nil.

(1) Suppose that $H$ and $K$ are both $A$ nil. There are no mixed $A$ nil groups [4, hilfssatz 9] so we may assume that $H$ is a torsion group, and that $K$ is torsion free. Let $R$ be an associative ring on $G$, such that $R$ is not a zeroring. Clearly $H$ is an ideal in $R$, and so $H=\langle h\rangle$. Let $\boldsymbol{m}=|\boldsymbol{h}|$, so $\boldsymbol{m} \boldsymbol{h}=0$. There are no nontrivial, bounded $\boldsymbol{A}$ nil-groups [3, Satz 1 and Zusatz], a contradiction.

(2) Suppose that $H=(x)$, and $K=(e)$ with $|x|=n<\infty$, and $|e|=$ $\infty$. Let $R$ be the ring on $G$ with multiplication induced by $x^{2}=x e=$ $e x=0, e^{2}=n e$. Clearly, $R$ is an associative ring on $G$, and $R$ is not a zeroring. Hence $R=\langle s x+t e\rangle, s, t \in Z$. Every $y \in R$ is of the form $y=m_{y} s x+\left(m_{y}+u_{y} n\right) t e, m_{y}, u_{y} \in Z$. In particular, $\quad\left(m_{e}+u_{e} n\right) t=1$. Hence $t= \pm 1$. Therefore $m_{x}+u_{x} n=0$, so that $n \mid m_{x}$. However $x=$ $m_{x} s x=0$, a contradiction.

THEOREM 3. Let $G$ be a torsion free, ASPI-group. Then $G$ is either indecomposable, or the direct sum of two A nil-groups. 
Proof. By Lemma 1, it suffices to negate that $G=$ $\left(x_{1}\right) \oplus\left(x_{2}\right), x_{i} \neq 0, i=1,2$. Suppose this is so. Let $R$ be the ring on $G$ with multiplication induced by

$$
x_{\imath} x_{\jmath}= \begin{cases}3 x_{i} & \text { if } i=j, i=1,2 \\ 0 & \text { if } i \neq j, i, j=1,2\end{cases}
$$

Then $R=\left\langle k_{1} x_{1}+k_{2} x_{2}\right\rangle, k_{1} \in Z, k_{i} \neq 0, i=1,2$. Every $x \in R$ is of the form

$$
x=\left(r_{x}+3 s_{x}\right) k_{1} x_{1}+\left(r_{x}+3 t_{x}\right) k_{2} x_{2}, r_{x}, s_{x}, t_{x} \in Z
$$

$r_{x_{1}}+3 s_{x_{1}}= \pm 1$, so that $r_{x_{1}} \equiv \pm 1(\bmod 3)$. However, $r_{x_{1}}+3 t_{x_{1}}=0$, so that $r_{x_{1}} \equiv 0(\bmod 3)$, a contradiction.

Corollary. Let $G$ be a torsion free SPI-group. Then $G$ is indecomposable.

Proof. Theorem 3, and the Corollary to Lemma 1.

The above Corollary yields that if $G$ is a torsion free $S P I$-group then either $G \simeq Q$, the group of rational numbers, or $G$ is reduced, [1, Theorem 21.3].

THEOREM 4. Let $G$ be a nonzero torsion group. The following are equivalent:

(1) $G$ is bounded.

(2) $G$ is an API-group.

(3) $G$ is a PI-group.

Proof. (1) $\Rightarrow(2)$ : Suppose that $n G=0, n$ a positive integer. Then $G \simeq \bigoplus_{p \mid n}\left[\bigoplus_{\alpha_{k}} Z\left(p^{k}\right)\right], p$ a prime with $p^{k} \mid n$, and $\alpha_{k}$ a cardinal number, [1, Theorem 17.3, and Theorem 8.4]. For each $p^{k} \mid n$, put $H_{p^{k}}=$ $\bigoplus_{\alpha_{k}} Z\left(p^{k}\right)$. Then $G \simeq \bigoplus_{p^{k} \mid n} H_{p^{k}}$ : There exists an associative unital PIring $R_{p^{k}}$ on $H_{p^{k}}$, for all $p^{k} \mid n$, [1, Lemma 122.3]. The ring direct sum $R=\bigoplus_{p^{k} \mid n} R_{p^{k}}$ is an $A P I$-ring on $G$ which is not the zeroring on $G$ [5, Chapt. 4, Theorem 33].

(2) $\Rightarrow$ (3): Obvious.

(3) $\Rightarrow(1)$ : Let $R$ be a $P I$-ring on $G$ which is not the zeroring on $G$. Then $R=\langle x\rangle$. Let $n=|x|$. Clearly, $n G=0$.

COROllary. Let $G$ be a mixed group. If $G$ is an $(A) P I$-group, then $T(G)$ (the torsion part of $G$ ) is bounded, and $T / T(G)$ is an (A)PI-group. Conversely, if $T(G)$ is bounded, and if there exists a unital (A)PI-ring on $G / T(G)$, then $G$ is $(A) P I$. 
Proof. Let $G$ be an $(A) P I$-group, and let $R$ be an $(A) P I$-ring on $G$ which is not a zeroring. Since $T(G)$ is an ideal in $R, T(G)=\langle x\rangle$, and $n T(G)=0, n=|x|$. Clearly $R / T(G)$ is an $(A) P I$-ring with identity on $G / T(G)$.

Suppose that $T(G)$ is bounded, and that there exists an $(A) P I$-ring with identity on $G / T(G)$. Then $G \simeq T(G) \oplus G / T(G)$, [1, Theorem 100.1]. There exists an $A P I$-ring $R_{1}$ with identity on $T(G)$, [1, Lemma 122.3]. Let $R_{2}$ be a unital $(A) P I$-ring on $G / T(G)$. Let $R$ be the ring direct sum $R=R_{1} \oplus R_{2}$, with $e_{i}$ the identity of $R_{\imath}, i=1,2$. Let $l$ be an ideal in $R$. Then $l=\left(l \cap R_{1}\right) \oplus\left(l \cap R_{2}\right)$. Since $l \cap R_{l}$ is an ideal in $R_{i}, l \cap R_{l}=\left\langle x_{i}\right\rangle, i=1,2$. Clearly, $\left\langle x_{1}+x_{2}\right\rangle \subseteq l$. However $\quad x_{i}=$ $e_{i}\left(x_{1}+x_{2}\right) \in\left\langle x_{1}+x_{2}\right\rangle$ for $i=1,2$. Hence $l=\left\langle x_{1}+x_{2}\right\rangle$.

Additional information concerning $P I$-groups and the classification of $\pi$-groups for other ring properties $\pi$ may be found in [2].

LeMmA 2. If a group $G$ is finitely generated, then $G$ is $S N$.

Proof. Obvious.

Lemma 3. Let a group $G=H \oplus K, H \neq 0, K \neq 0$, be $S N$. Then either $G$ is finitely generated, or $H$ and $K$ are both nil.

Proof. Suppose that $H$ is not nil. Let $S$ be a nonzeroring on $H$, and let $T$ be the zeroring on $K$. The ring direct sum $R=S \oplus T$ is a ring on $G$ which is not the zeroring, with ideal $T$. Let $t_{1}, \cdots, t_{n}$ be a finite set of generators for $T$. Then $K=\left(t_{1}, \cdots, t_{n}\right)$. This implies that $K$ is not nil. The same argument, interchanging the roles of $H$ and $K$, yields that $H$ is finitely generated. Hence $G$ is finitely generated.

THEOREM 5. Let $G$ be a non torsion free group. $G$ is $S N$ if and only if $G$ is finitely generated.

Proof. By Lemma 2, it suffices to show that if $G$ is non torsion free and $S N$, then $G$ is finitely generated.

(1) Suppose that $G$ is a torsion group. If $G$ is indecomposable then $G$ is cyclic [1, Corollary 27.4 and Theorem 120.3]. We may assume, by Lemma 3, that $G=H \oplus K, H \neq 0, K \neq 0$, with $H$ and $K$ both nil. This implies that $G$ is nil [1, Theorem 120.3]. A contradiction.

(2) Suppose that $G$ is a mixed group. Then $G$ is decomposable [1, Corollary 27.3]. By Lemma 3, it suffices to negate that $G=$ $H \oplus K, H \neq 0, K \neq 0$, with $H$ and $K$ both nil. Suppose that this is so. By [1, Theorem 120.3] we may assume that $H$ is a torsion group and that $K$ is torsion free. However, by [1, Proposition 126.2] $H$ is bounded and hence not nil, a contradiction. 
Corollary. Let $G$ be an $S N$ group. Then $T(G)$ and $G / T(G)$ are $S N$.

Proof. If $G$ is torsion free then the statement is trivial. Otherwise, $G$ is finitely generated by Theorem 5 , and so $T(G)$ and $G / T(G)$ are $S N$ by Lemma 2 .

A torsion-free $S N$-group need not be finitely generated; e.g. $Q$ the group of rational numbers. However, we have the following:

THEOREM 6. Let $G$ be an $S N$-group. Then $G$ is either indecomposable, or finitely generated.

Proof. By Lemma 3, it suffices to negate that $G=$ $H \oplus K, H \neq 0, K \neq 0$, with $H$ and $K$ both nil. Suppose this is so. Let $R$ be a ring on $G$ which is not a zeroring. Then $R=\left\langle x_{1}, \cdots, x_{n}\right\rangle, n$ a positive integer. Put $x_{i}=h_{i}+k_{i}, h_{i} \in H, k_{i} \in K, 1 \leqq i \leqq n$.

(1) Suppose that $R^{2} \subseteq K$. Let $h \in H$. Since $h \in R, h=$ $\sum_{l=1}^{n} r_{i}\left(h_{i}+k_{\imath}\right)+x, r_{i} \in Z, 1 \leqq i \leqq n, x \in R^{2}$. However $R^{2} \subseteq K$. Hence $h=\sum_{i=1}^{n} r_{i} h_{i}$, and $H$ is finitely generated. This contradicts the fact that $H$ is nil.

(2) Suppose that $R^{2} \notin K$. For $g_{i}, g_{2} \in G$ define $g_{1} \times g_{2}=$ $\pi_{H}\left(g_{1} g_{2}\right)$. Then $S=(G, \times)$ is a ring on $G$ which is not a zeroring satisfying $S^{2} \subseteq H$. The above argument yields that $K$ is finitely generated, contradicting the fact that $K$ is nil.

\section{REFERENCES}

1. L. Fuchs, Infinite Abelian Groups, Academic Press, New York and London, vol. 1 (1970), vol. 2 (1973).

2. Z. Schlussel, On Additive Groups of Rings, Master's thesis, Bar-Ilan University, 1976 (Hebrew with English summary).

3. T. Szele, Zur theorie der zeroringe, Math. Annal., 121 (1949), 242-248.

4. — Gruppentheoretische beziehungen bei gewissen ringkonstruktionen, Math. Zeitschr., 54 (1951), 168-180.

5. O. Zariski and P. Samuel, Commutative Algebra, vol. 1, Van-Nostrand, Princeton (1958).

Received December 28, 1976 and in revised form June 13, 1977.

BAR-ILAN UNIVERSITY

RAMAT-GAN, ISRAEL 


\section{PACIFIC JOURNAL OF MATHEMATICS EDITORS}

ICHARD ARENS (Managing Editor)

niversity of California

os Angeles, CA 90024

\section{. A. BEAUmont}

niversity of Washington

sattle, WA 98105

. C. MOORE

niversity of California

erkeley, CA 94720
J. DUGUNDJI

Department of Mathematics

University of Southern California

Los Angeles, CA 90007

R. FINN AND J. MiLgRAM

Stanford University

Stanford, CA 94305

\section{ASSOCIATE EDITORS}
F. BECKENBACH
B. H. NEUMANN
F. WOLF
K. YosHidA

\section{SUPPORTING INSTITUTIONS}

NIVERSITY OF BRITISH COLUMBIA

UNIVERSITY OF SOUTHERN CALIFORNIA

ALIFORNIA INSTITUTE OF TECHNOLOGY STANFORD UNIVERSITY

NIVERSITY OF CALIFORNIA

ONTANA STATE UNIVERSITY

UNIVERSITY OF HAWAII

NIVERSITY OF NEVADA

UNIVERSITY OF TOKYO

EW MEXICO STATE UNIVERSITY

UNIVERSITY OF UTAH

REGON STATE UNIVERSITY

NIVERSITY OF OREGON

WASHINGTON STATE UNIVERSITY

UNIVERSITY OF WASHINGTON

SAKA UNIVERSITY

AMERICAN MATHEMATICAL SOCIETY

The Supporting Institutions listed above contribute to the cost of publication of this Journal, but they a t owners or publishers and have no responsibility for its contents or policies.

Mathematical papers intended for publication in the Pacific Journal of Mathematics should be in typ Irm or offset-reproduced (not dittoed), double spaced with large margins. Underline Greek letters in re ierman in green, and script in blue. The first paragraph or two must be capable of being used separately as 'nopsis of the entire paper. Items of the bibliography should not be cited there unless absolutely necessary, hich case they must be identified by author and Journal, rather than by item number. Manuscripts, uplicate, may be sent to any one of the four editors. Please classify according to the scheme of Math. Revieu Idex to Vol. 39. All other communications should be addressed to the managing editor, or Elaine Bart Iniversity of California, Los Angeles, California, 90024.

100 reprints are provided free for each article, only if page charges have been substantially pai dditional copies may be obtained at cost in multiples of 50 .

The Pacific Journal of Mathematics is issued monthly as of January 1966. Regular subscription rate: \$72. year (6 Vols., 12 issues). Special rate: $\$ 36.00$ a year to individual members of supporting institutions.

Subscriptions, orders for numbers issued in the last three calendar years, and changes of address should ent to Pacific Journal of Mathematics, 103 Highland Boulevard, Berkeley, California, 94708.

UBLISHED BY PACIFIC JOURNAL OF MATHEMATICS, A NON-PROFIT CORPORATION

Printed at Jerusalem Academic Press, POB 2390, Jerusalem, Israel.

Copyright (C) 1978 Pacific Journal of Mathematics

All Rights Reserved 


\section{Pacific Journal of Mathematics}

\section{Vol. 75, No. $1 \quad$ September, 1978}

Mieczyslaw Altman, General solvability theorems

Denise Amar and Eric Amar, Sur les suites d'interpolation en plusieurs variables ..........................................

Herbert Stanley Bear, Jr. and Gerald Norman Hile, Algebras which satisfy a second order linear partial differential equation ..................

Marilyn Breen, Sets in $R^{d}$ having $(d-2)$-dimensional kernels ............

Gavin Brown and William Moran, Analytic discs in the maximal ideal space

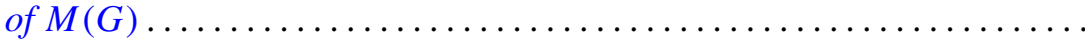

Ronald P. Brown, Quadratic forms with prescribed Stiefel-Whitney

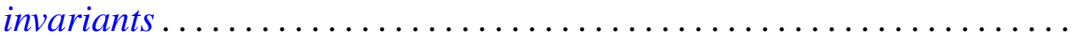

Gulbank D. Chakerian and H. Groemer, On coverings of Euclidean space by convex sets

S. Feigelstock and Z. Schlussel, Principal ideal and Noetherian groups.....

Ralph S. Freese and James Bryant Nation, Projective lattices ............

Harry Gingold, Uniqueness of linear boundary value problems for

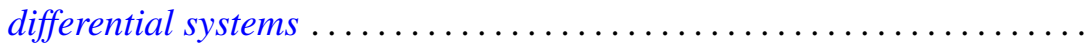

John R. Hedstrom and Evan Green Houston, Jr., Pseudo-valuation domains. . .

William Josephson, Coallocation between lattices with applications to measure extensions

M. Koskela, A characterization of non-negative matrix operators on $l^{p}$ to $l^{q}$ with $\infty>p \geq q>1$

Kurt Kreith and Charles Andrew Swanson, Conjugate points for nonlinear differential equations...........................

Shoji Kyuno, On prime gamma rings ........................ 185

Alois Andreas Lechicki, On bounded and subcontinuous multifunctions ..

Roberto Longo, A simple proof of the existence of modular automorphisms in approximately finite-dimensional von Neumann algebras ...

Kenneth Millett, Obstructions to pseudoisotopy implying isotopy for

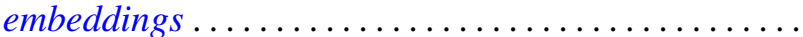

William F. Moss and John Piepenbrink, Positive solutions of elliptic equations. .

Mitsuru Nakai and Leo Sario, Duffin's function and Hadamard's

conjecture

Mohan S. Putcha, Word equations in some geometric semigroups ...

Walter Rudin, Peak-interpolation sets of class $C^{1} \ldots \ldots \ldots$

Elias Saab, On the Radon-Nikodým property in a class of locally convex

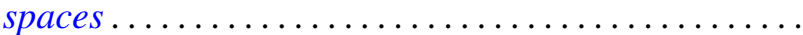

